\title{
ARTICLE OPEN Mesenchyme-free expansion and transplantation of adult alveolar progenitor cells: steps toward cell-based regenerative therapies
}

Aaron I. Weiner ${ }^{1,2,3}$, Sergio R. Jackson ${ }^{1,2,3}$, Gan Zhao ${ }^{1,2,3}$, Kwaku K. Quansah ${ }^{1,2,3}$, Joseph N. Farshchian ${ }^{1}$, Katherine M. Neupauer ${ }^{1}$, Elizabeth Q. Littauer ${ }^{4}$, Andrew J. Paris ${ }^{5}$, Derek C. Liberti $\mathbb{B D}^{2,3,6,7}$, G. Scott Worthen ${ }^{3,8}$, Edward E. Morrisey ${ }^{2,3,6,7}$ and Andrew E. Vaughan (iD ${ }^{1,2,3}$

Alveolar type-2 (AT2) cells are necessary for the lung's regenerative response to epithelial insults such as influenza. However, current methods to expand these cells rely on mesenchymal co-culture, complicating the possibility of transplantation following acute injury. Here we developed several mesenchyme-free culture conditions that promote growth of murine AT2 organoids. Transplanting dissociated AT2 organoids into influenza-infected mice demonstrated that organoids engraft and either proliferate as AT2 cells or unexpectedly adopt a basal cell-like fate associated with maladaptive regeneration. Alternatively, transplanted primary AT2 cells also robustly engraft, maintaining their AT2 lineage while replenishing the alveolar type-1 (AT1) cell population in the epithelium. Importantly, pulse oximetry revealed significant increase in blood-oxygen saturation in primary AT2 recipients, indicating that transplanted cells also confer increased pulmonary function after influenza. We further demonstrated that both acid installation and bleomycin injury models are also amenable to AT2 transplantation. These studies provide additional methods to study AT2 progenitor potential, while serving as proof-of-principle for adoptive transfer of alveolar progenitors in potential therapeutic applications.

npj Regenerative Medicine (2019)4:17; https://doi.org/10.1038/s41536-019-0080-9

\section{INTRODUCTION}

The lung is able to regenerate following severe injury such as influenza due to resident alveolar type-2 (AT2) cells within the alveoli. AT2 cells can self-renew and differentiate into alveolar type1 (AT1) cells, thus producing essential surfactant and providing a source of oxygen-exchanging epithelial AT1 cells. ${ }^{1,2}$ However, this response is imperfect and the lung also exhibits aberrant tissue remodeling that results in the appearance of ectopic cell types in the alveoli, especially bronchiolized epithelium ${ }^{3}$ containing solitary chemosensory/tuft cells. ${ }^{4}$ This dysplastic tissue arises largely due to expansion and occupation of the alveoli by $\mathrm{p} 63^{+} \mathrm{Krt} 5^{+}$cells derived from migratory basal-like "lineage-negative" cell populations. ${ }^{3,5-7}$ Initially described in mice, there is increasing evidence that this dysplastic response also occurs in humans and may impair longterm functional recovery. ${ }^{8-10}$

Here we describe two methods to promote lung regeneration after influenza infection: transplantation of AT2 organoids grown in defined culture conditions and transplantation of primary AT2 cells (Supplementary Fig. S1). Established methods for AT2 organoids require co-culture with lung mesenchyme, ${ }^{2,11}$ of which the mesenchymal alveolar niche cell (MANC) subpopulation primarily supports AT2 self-renewal in vivo and in vitro. ${ }^{12}$ Despite its benefits for ex vivo AT2 maintenance, lung mesenchyme co- culture is an undesirable system for subsequent transplantation, as separation of the intermingled cell types in vitro is technically challenging and transplantation of mesenchyme can exacerbate organ repair and fibrosis following injury. ${ }^{13-15}$ Specifically, fibroblast-like cells present in mesenchymal co-culture conditions are predominant sources of, and responders to, transforming growth factor- $\beta$ and other fibrosis-inducing growth factors and cytokines. ${ }^{16-19}$ In addition, the pro-AT2 signals secreted by the lung mesenchyme vary in concentration and composition, making lung mesenchyme co-culture disadvantageous for our goal of defining precise ex vivo AT2 growth conditions. The AT2 culture methods described here allow for well-defined in vitro expansion of AT2 organoids, while abrogating the need for supporting mesenchymal cells, permitting organoid transplantation. Primary AT2 cells were also transplanted, responding similarly to native AT2 progenitors by proliferating and differentiating into AT1 cells. Importantly, primary AT2 transplant measurably improved pulmonary function as indicated by faster recovery of blood-oxygen saturation. This report provides techniques that can be leveraged into future therapeutic approaches in lung regeneration.

\footnotetext{
${ }^{1}$ Department of Biomedical Sciences, School of Veterinary Medicine, University of Pennsylvania, Philadelphia, PA 19104 , USA; ${ }^{2}$ Institute for Regenerative Medicine, University of Pennsylvania, Philadelphia, PA 19104, USA; ${ }^{3}$ Penn Center for Pulmonary Biology, University of Pennsylvania, Philadelphia, PA 19104, USA; ${ }^{4}$ Department of Microbiology, Perelman School of Medicine, University of Pennsylvania, Philadelphia, PA 19104, USA; ${ }^{5}$ Pulmonary, Allergy and Critical Care Division, Department of Medicine, Perelman School of Medicine, University of Pennsylvania, Philadelphia, PA 19104, USA; ${ }^{6}$ Department of Cell and Developmental Biology, Perelman School of Medicine, University of Pennsylvania, Philadelphia, PA 19104, USA; ${ }^{7}$ Penn Cardiovascular Institute, University of Pennsylvania, Philadelphia, PA 19104, USA and ${ }^{8}$ Division of Neonatology, Department of Pediatrics, Perelman School of Medicine, University of Pennsylvania, Philadelphia, PA 19104, USA

Correspondence: Andrew E. Vaughan (andrewva@vet.upenn.edu)
}

Received: 25 January 2019 Accepted: 1 August 2019

Published online: 20 August 2019 


\section{RESULTS}

We initially asked whether utilizing growth factors and modulators of signaling pathways involved in lung development might allow for culture of pure AT2 cells, removing the need for mesenchymal support. AT2 cells were flow sorted using an established gating scheme $^{20}$ (Fig. 1a and Supplementary Fig. 2). Cytospins validated this strategy, yielding a $96.25 \pm 0.47 \%$ pure AT2 population (Fig. $1 \mathrm{~b}, \mathrm{c})$, which we additionally confirmed by sorting from a tamoxifen-treated SPC-CreERT2(tdTomato) reporter mouse in which $96.4 \%$ of gated cells were lineage-labeled AT2s (Fig. 1d). Capitalizing on incorporation of developmental signals such as Wnt, fibroblast growth factor (FGF), and bone morphogenetic protein (BMP) signaling, we modified existing culture conditions $s^{3,6}$ to promote mesenchyme-free growth of purified AT2 cells. Eleven culture conditions were tested (C1-C11), in addition to a serumfree condition containing all growth factors (C12) and a mesenchymal co-culture condition $(\mathrm{C} 1+\mathrm{M})$ (Table 1). The lung mesenchyme population for $\mathrm{C} 1+\mathrm{M}$ was isolated by a $\mathrm{CD} 45^{-}$ $\mathrm{PECAM}^{-} \mathrm{EpCAM}^{-}$sorting strategy (Supplementary Fig. 3). This population consisted largely of Pdgfra $^{+}(\sim 53 \%$ of sorted lung mesenchyme) cells, enriched in MANCs, and Wnt2 ${ }^{+}(\sim 6 \%$ of sorted lung mesenchyme) cells, as well as aSMA ${ }^{+}$airway smooth muscle cells and/or myofibroblasts ( $4 \%$ of sorted lung mesenchyme) (Supplementary Fig. 3). AT2 cells grew into spherical organoids after 13 days in culture (Fig. 1e). Immunostaining displayed expression of canonical AT2 markers such as surfactant protein C (SPC) and Lamp3 (Fig. 1f), and quantitative PCR (qPCR) confirmed that most conditions maintained expression levels of SPC comparable to freshly isolated (FI) AT2 cells (Fig. 1h). However, some conditions showed slightly higher expression of Scgb3a2, an airway cell marker, and cytokeratin 5 (Krt5), an indicator of lung dysplasia (Fig. 1i, j). Diameter was used to assess proliferative ability and overall health (Fig. 1g). In accordance with established methods, ${ }^{2}$ mesenchymal co-culture generated the largest relative organoids. Predictably, conditions containing all or most growth factors generated the largest spheroids (Supplementary Fig. 4a-c).

Utilizing two promising conditions, C2 and C12, AT2 organoids were grown, dissociated, and transplanted into influenza-injured recipient mice 11 days post infection (DPI) as previously described. ${ }^{3}$ Immunostaining of recipient lungs 13 days post transplant (DPT, $24 \mathrm{DPI})$ revealed that engraftments could adopt two distinct fates (Fig. 2a): maintenance of the AT2 lineage (SPC and Lamp3 expression) (Fig. 2e, f) or, surprisingly, expression of markers of dysplastic regeneration (Scgb3a2 and Krt5) (Fig. 2d) despite low expression of these genes in culture. These expression patterns were mutually exclusive; dysplastic engraftments never expressed AT2 markers (Fig. 2b, c) and vice versa (Fig. 2g). Pulse oximetry of organoid transplant recipients was not significantly different than that of mock-transplanted mice (Fig. 2h). Together, these data indicate that although sorted AT2 cells can be expanded in mesenchyme-free culture conditions without disrupting their AT2 fate, these organoids can spontaneously adopt a dysplastic fate in vivo.

Given the unexpected expression of dysplastic markers upon organoid transplant, we reasoned that primary AT2 cells, which never experience in vitro conditions, may retain more appropriate lineage restriction upon transplant. Indeed, primary AT2 cell transplants robustly engraft and expand throughout a significant portion of influenza-injured lobes (Fig. 3a). Immunostaining of recipient lungs 13 DPT revealed that all engrafted primary AT2 cells either remain as AT2 cells (Fig. 3b, c) or differentiate into AT1 cells (Fig. 3d and Supplementary Fig. 5a, b). In contrast with organoid engraftments (Supplementary Fig. 6c), none of the primary cell engraftments express Scgb3a2 or Krt5 (Fig. 3e and Supplementary Fig. 6a, b). Primary AT2 cells were also transplanted into an array of other injury models to determine whether the extent or type of damage influenced the ability of primary
AT2s to engraft. Robust engraftments were observed in 4/4 acidinjured and 4/4 bleomycin-injured mice at 13 DPT (14 days post acid instillation and 23 days post bleomycin administration, respectively). These engraftments exclusively contributed to AT2 and AT1 regeneration similarly to influenza-injured recipients and never exhibited dysplastic regeneration markers (Fig. 4a-f). Interestingly, primary AT2 transplants into Streptococcus pneumonia $(S p)$-infected recipients resulted in only rare engraftments consisting of $1-3$ cells (Fig. $4 \mathrm{~g}, \mathrm{~h}$ ). These results suggest that primary AT2 cells are capable of engrafting into a diverse array of pulmonary injury models, properly maintain their fate and in vivo function, and aid in re-epithelializing the damaged lung.

Strikingly, pulse oximetry revealed that blood-oxygen saturation was significantly higher 12 DPT (23 DPI) in influenza-injured recipient mice compared with that of mock-transplanted controls $(p=0.0315)$ (Fig. 5a, b). Linear regression analysis also indicated that the slope of blood-oxygen saturation change over time for transplante recipient mice was very nearly statistically significantly higher $(p=0.0581)$ than that of control mice (Fig. $5 c)$, indicating that primary cell-transplanted mice recover $\sim 65 \%$ faster than controls. We conclude that primary AT2 cells are capable of engrafting into injured murine lungs, serving their role as stem cells by partially replenishing the AT1 and AT2 cell populations and aiding in restoration of pulmonary function.

\section{DISCUSSION}

This report provides methods to expand and transplant AT2 cells. Factors that promote branching morphogenesis and AT2 expansion such as FGF10, $\mathrm{KGF}^{21}$ and $\mathrm{Wnt}^{22}$ proved sufficient to expand AT2 organoids without mesenchymal co-culture, yielding large organoids with high SPC expression. Interestingly, we saw a stepwise decline in organoid diameter with each growth factor removed from conditions $C 2$ to $C 7$, suggesting an additive effect of each factor. This approach failed to reveal a single growth factor that was absolutely required for maintaining either SPC expression or proliferative ability. Organoid diameter in mesenchyme-free culture conditions approached but never matched that of coculture, suggesting that additional factors will be necessary to maximize organoid size, or that some growth signals are derived via direct cell-cell interactions. Similarly, organoid SPC levels did not reach those of uncultured AT2 cells, suggesting that further optimization is possible.

AT2 organoids engraft in vivo without the use of complex delivery or expansion systems, many retaining their AT2 fate. Despite this, organoids sometimes adopt a dysplastic fate upon engraftment, a surprising finding given that definitive lineage tracing has demonstrated that $\mathrm{Krt}^{+}$cells are never derived from endogenous AT2 cells in vivo. ${ }^{3}$ Considering negligible expression of dysplastic markers in vitro (Fig. 1i, j), differentiation into maladaptive fates could result from unintentional "priming" in vitro that manifests in vivo. Transplantation of AT2 organoids did not appear to increase the oxygen-exchange capability of injured recipient mice, likely to be due to the contribution of engraftments to non-oxygen-exchanging dysplastic tissue. Further elucidation of the transcriptional changes that occur in AT2 organoids and the signals encountered by transplanted organoids in the influenza-injured microenvironment/engraftment niche should provide insight into how to further optimize organoid transplant.

In contrast to organoid transplants, primary cell engraftments either expande as AT2 cells or differentiate into AT1 cells, indicating that transplanted adult AT2s have the capability to robustly differentiate into AT1 cells in vivo after a wide array of pulmonary insults, analogously to their native post-flu counterparts. Retention of solely alveolar markers in primary cell engraftments further indicates that in vitro culturing is likely responsible for dysplastic organoid differentiation. Interestingly, 
A

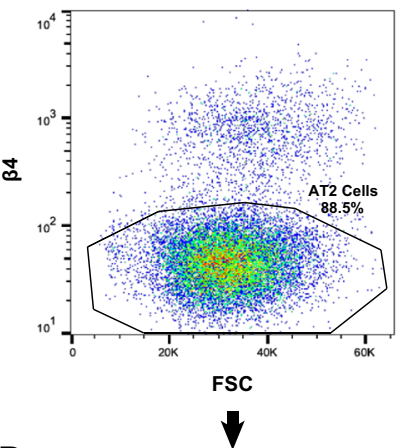

D

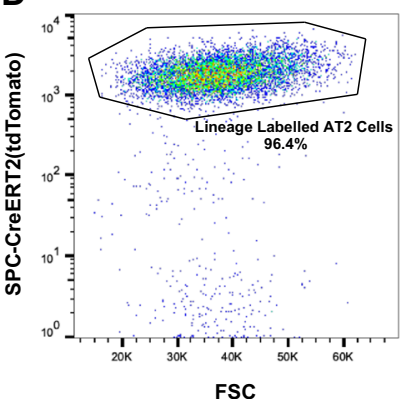

B
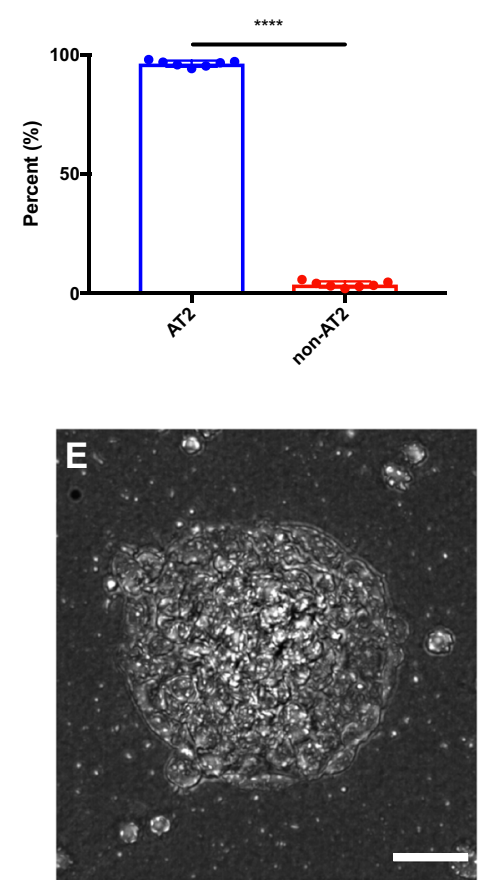
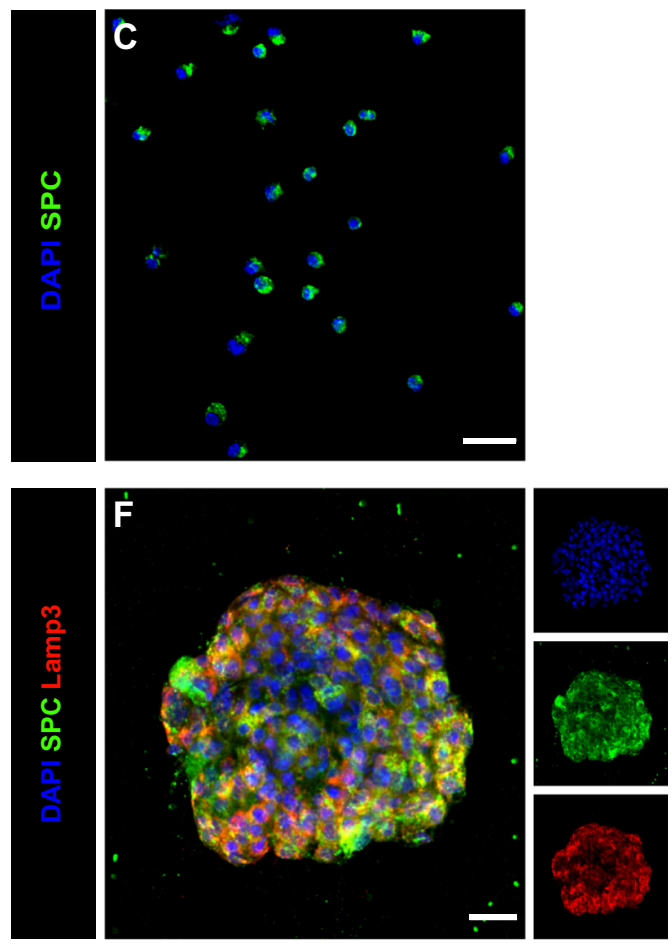
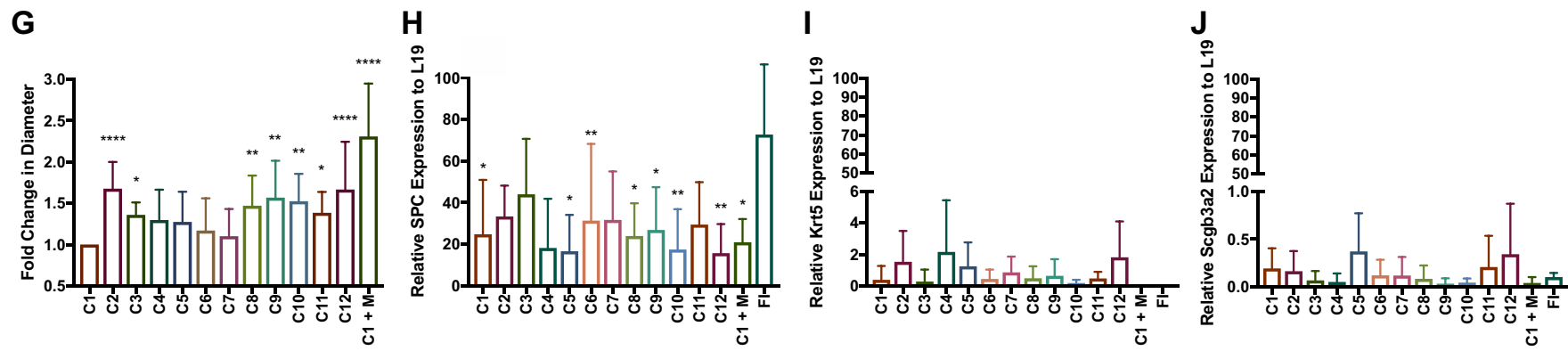

Fig. 1 Mesenchyme-free culture conditions generate healthy AT2 organoids. a FACS isolation of AT2 cells by gating on $\beta 4^{-}$lung epithelial cells. b AT2-sorted purity quantification by manual cell count of cytospins yields a $96.25 \pm 0.47 \%$ pure population. $n=7$ from 7 independent experiments, mean \pm SEM, ${ }^{* * *} p<0.0001$ by two-tailed Welch's $t$-test. c Representative immunofluoresence image of an AT2 cytospin used for purity checks. Scale bar $=25 \mu \mathrm{m}$. d Validation of gating scheme and purity by subgating $\beta 4^{-}$epithelial cells from a tamoxifen-administered SPC-CreERT2(tdTomato) reporter mouse. $96.4 \%$ of $\beta 4^{-}$cells were lineage-traced, similar to cytospin purity quantification. e, f Representative bright-field max projection and immunofluorescence images of cytospun AT2 organoids grown in C2 for 9 days. Scale bar $=25 \mu \mathrm{m}$. $\mathbf{g}$ Change in organoid diameter between culture conditions, normalized to the average diameter of $\mathrm{C} 1$ organoids. Significance tests are relative to $\mathrm{C} 1$. $\mathbf{h}-$ j qPCR shows that many culture conditions maintain high SPC expression (h), whereas expression of Krt5 (i) and Scgb3a2 (j) remain low across all conditions. Significance tests are relative to freshly isolated (FI) AT2 expression of corresponding genes. Data for $\mathbf{g}-\mathbf{j}$ are based on $n \geq 3$ for all conditions from at least 15 independent experiments and error bars represent SD

only small clusters of a few AT2s from primary AT2 transplants were found in the lungs of $S p$-infected recipient mice, in stark contrast to the widespread donor AT2 expansion found in other injury model recipients. This could be due to the presence of active infectious bacteria in the post-Sp lung at the time of transplant, ${ }^{23}$ whereas the other injury models used had either cleared the infection by the time of transplant or did not use infectious agents. Further studies will be needed to optimize the timing of adoptive AT2 transfer and to examine the possibility of transplant during bouts of active infection.

Pulse oximetry confirmed that transplanted primary AT2s assist in restoring the oxygen-exchange capacity of the epithelium, improving pulmonary function. The upward trend in oxygen saturation becomes statistically significant at 12 DPT in transplant recipients, demonstrating that primary AT2 transplantation confers a true restorative advantage at a relatively early time point in recovery. It remains to be determined whether functional benefits of cell transplant are mediated mostly by restoration of gas- exchanging AT1 cells, supplementation of surfactant production, or, likely, a combination of both. Long-term studies will be necessary to assess the longevity of transplanted primary cells and determine the ultimate extent to which they restore pulmonary function.

Orthotopic transplantation of adult progenitor cells and induced pluripotent stem cells (iPSCs) has been employed to restore physiological function in other organ systems. Transplantation of adult human hepatocytes and hepatic iPSC-derived organ buds into a mouse model of liver failure rescues this lethal genetic phenotype, with active in vivo contribution of the engrafted cells to albumin synthesis and drug metabolism. ${ }^{24,25}$ Similar experiments have been performed in the mammary gland, in which transplantation of a single mammary stem cell into cleared murine mammary fat pads is able to fully reconstitute a functional gland with milk-producing capability. ${ }^{26}$ Fetal murine enteric progenitors ${ }^{27}$ and human colon organoids ${ }^{28}$ are also capable of regenerating full intestinal crypts and villi following 
Table 1. Contents and concentration of growth factors and cell culture supplements used in each AT2 organoid culture condition

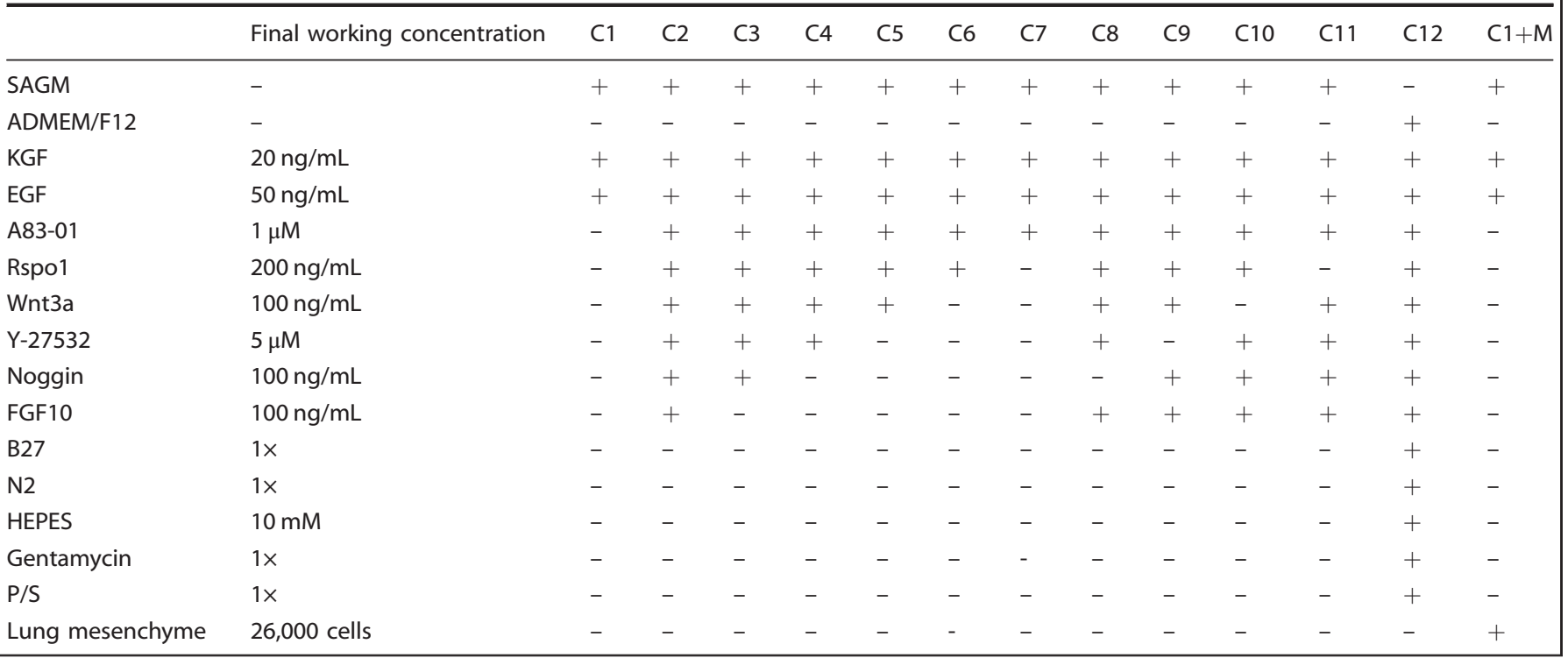

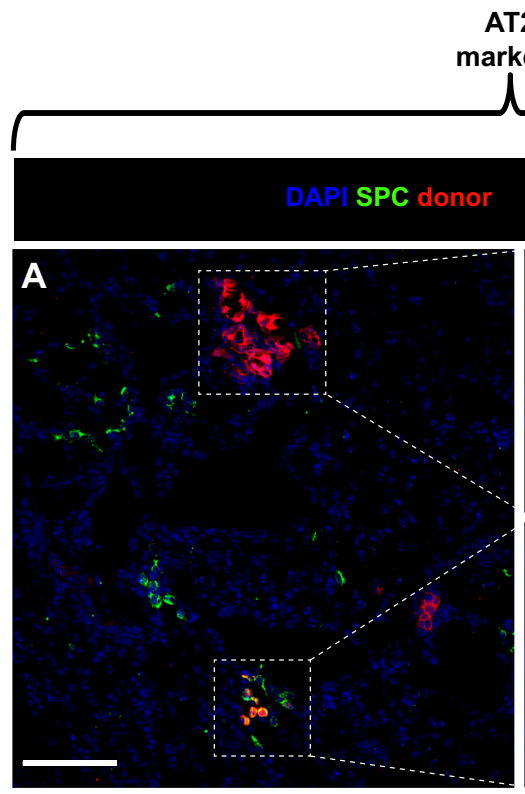

T2

markers
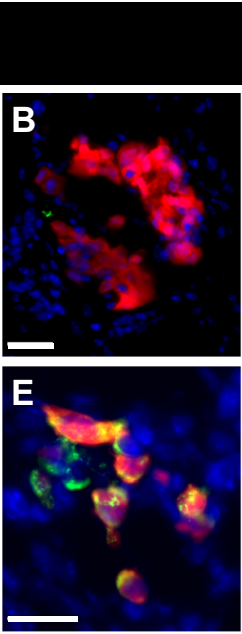

Dysplastic

markers

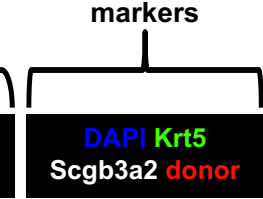

Lamp3 donor
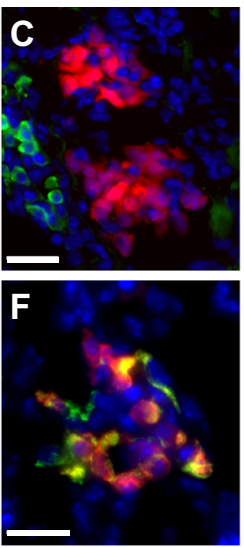

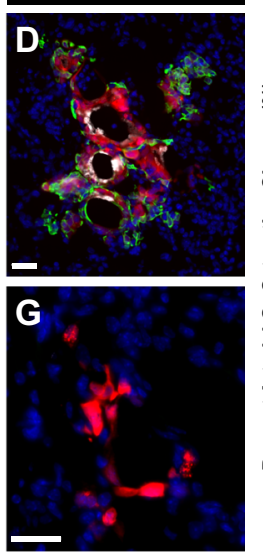

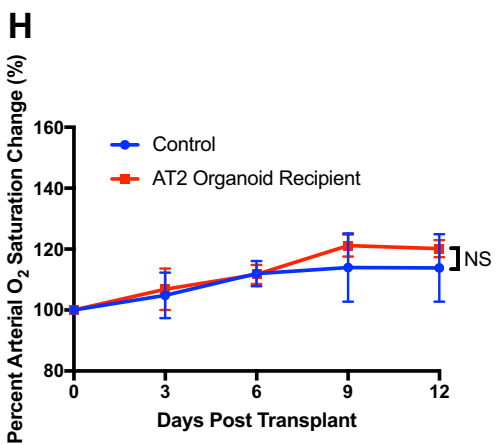

Fig. 2 AT2 organoids display a bipotential fate upon post-injury transplantation. a Immunofluorescence of injured recipient lungs receiving 50,000-100,000 AT2 organoid cells reveals proximate engraftments with differing cell fates. Scale bar $=100 \mu \mathrm{m}$. b-g Representative images of AT2 organoid engraftments. Some AT2 organoid engraftments adopt a maladaptive fate, exclusively expressing the dysplastic markers Krt5 and Scgb3a2 (d) as opposed to markers of their original AT2 fate (SPC and Lamp3) (b, c). Other engraftments remain as AT2 cells and maintain AT2 marker expression $(\mathbf{e}, \mathbf{f})$ without expressing dysplastic markers $(\mathbf{g})$. Immunostaining expression patterns were observed in five mice from three independent experiments. Scale bars $=25 \mu \mathrm{m}$. h Pulse oximetry readings were taken from 100,000 AT2 organoid cell-transplanted and mock-transplanted mice over the course of 12 days post transplant. No significant increase in $\% \mathrm{O}_{2}$ was detected. Data for $\mathbf{h}$ is based on control $n=7$ mice, transplanted $n=3$ mice from three independent experiments, and error bars represent SD. NS $\geq 0.05,{ }^{*} p<0.05,{ }^{* *} p<0.01$, ${ }^{* * *} p<0.001,{ }^{* * * *} p<0.0001$ by ordinary one-way ANOVA

dextran sulfate sodium-induced ulcerative colitis and de-epithelialization, respectively, although the extent to which these engraftments contribute to intestinal function has yet to be determined. Few studies have attempted epithelial progenitor cell transplant in the lung and fewer yet have assessed the functional outcomes of cell transplant. Transplantation of airway-derived progenitor cells, as opposed to AT2s, into the alveoli of influenza- injured mice has been performed by different groups, ${ }^{3,5}$ although studies of the resulting engraftments primarily focus on their in vivo cell-fate outcomes. In a creative approach, one group recently transplanted AT2 cells that underwent "interrupted reprogramming" via transient expression of the pluripotency factors into bleomycin-injured mice, improving metrics of tissue resistance and compliance, while seemingly helping to mitigate 

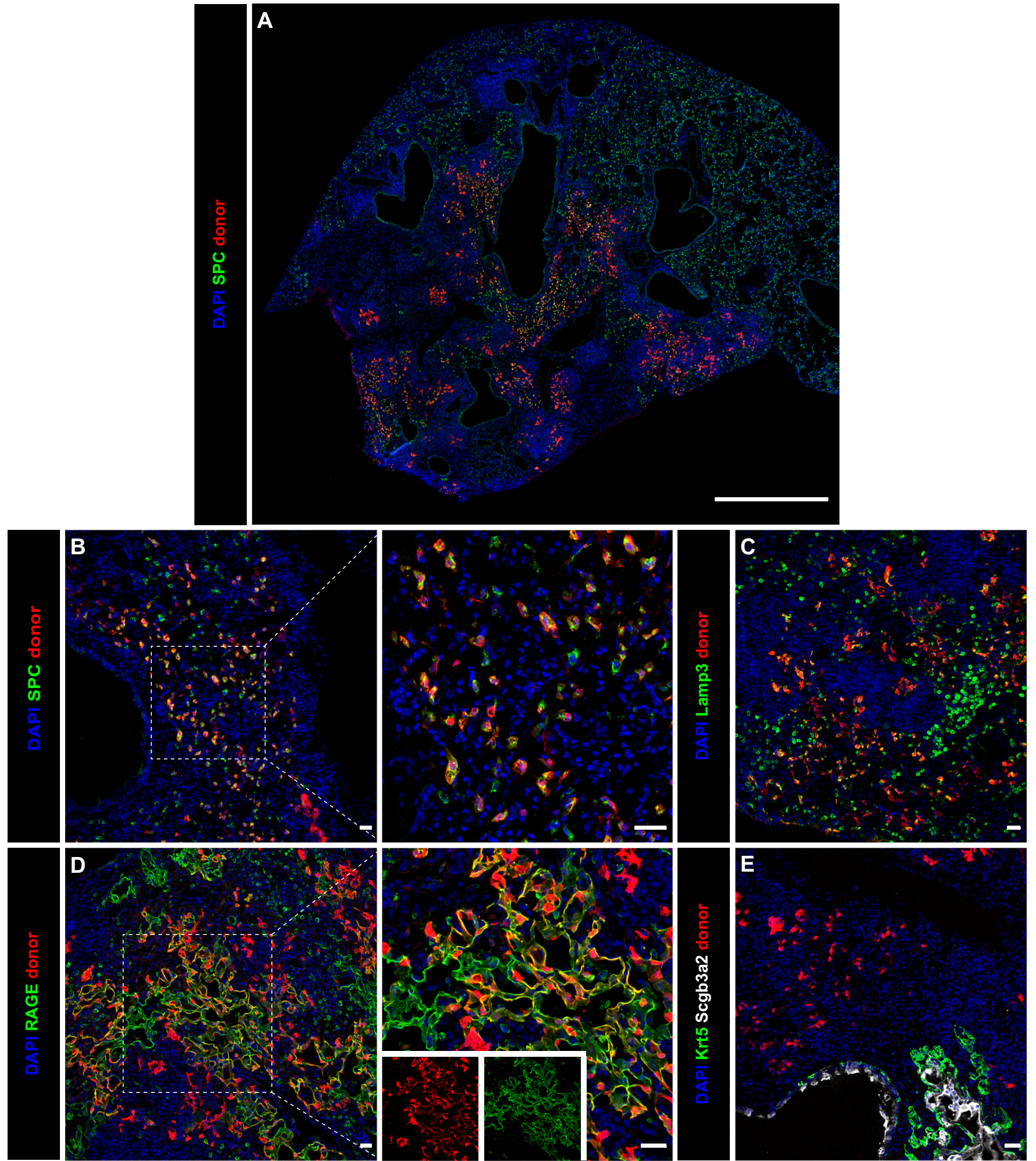

Fig. 3 Primary adult AT2 cell engraftments exclusively maintain an AT2 cell fate in vivo and contribute to re-epithelialization of the injured lung. a Whole-lobe immunostain of a primary AT2 transplant recipient, showing extent of engraftment and proliferation throughout injured lobes. Scale bar $=1000 \mu \mathrm{m}$. b-g Representative immunostains of AT2, AT1, and dysplastic markers demonstrating the fate of engrafted primary AT2 cells. Transplanted primary AT2 cells either expand as AT2 cells, evidenced by SPC (b) and Lamp3 (c) expression, or differentiate into AT1 cells expressing RAGE (d). Engraftments expand into zones exhibiting dysplasia but never express the dysplastic markers Krt5 and Scgb3a2 (e). Insets in $\mathbf{b}$ and $\mathbf{d}$ are of dashed boxes in larger image to highlight SPC and RAGE expression. Immunostaining expression patterns were observed in six mice from two independent experiments. Scale bars $=25 \mu \mathrm{m}$ 


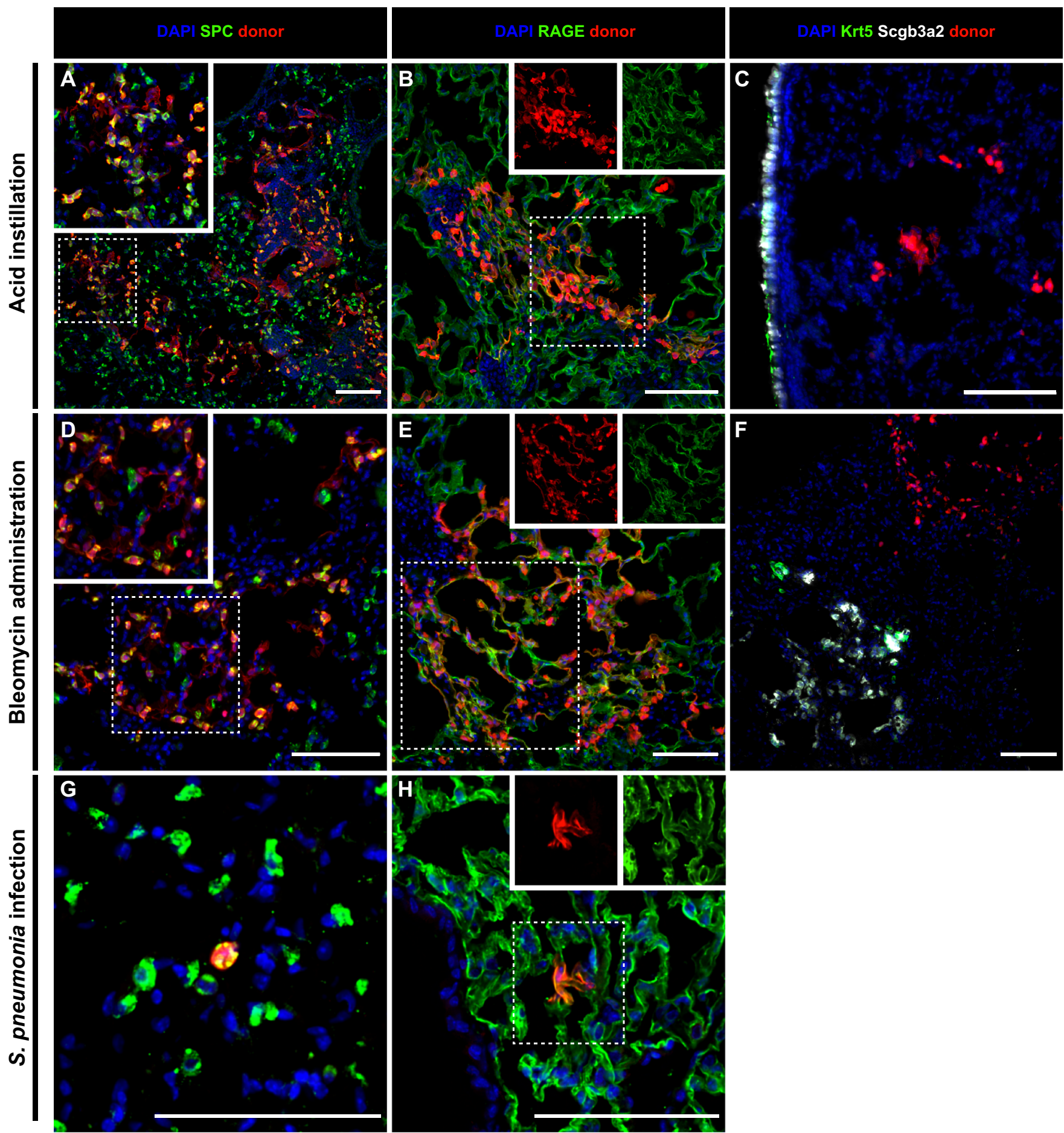

Fig. 4 Primary AT2 transplants into alternative injury models. Primary AT2 cells were transplanted into acid- (a-c), bleomycin- (d-f), and Spinjured $(\mathbf{g}, \mathbf{h})$ recipients. Transplanted AT2s engraft and expand exclusively as AT2s $(\mathbf{a}, \mathbf{d}, \mathbf{g})$ or AT1s (b, e, $\mathbf{h})$ in all injury models and never contributed to dysplasia (c, f). Magnified sections and individual channels are derived from dashed boxes to highlight individual cell/marker expression. Scale bars $=100 \mu \mathrm{m}$

fibrosis. ${ }^{29}$ Our study similarly finds that orthotopic transplantation of mature AT2 cells can directly contribute to restoration of the most critical pulmonary function, gas exchange.

Although further optimization of culture conditions to retain normal cell-fate restriction is still required, transplantation of primary AT2 cells demonstrated significant improvements in lung function recovery. The strict retention of alveolar fate and expansion in harsh injury environments of primary AT2s highlights the robustness of this progenitor population and underscores its utility as a source of progenitors for syngeneic transplant of alveolar epithelial cells following pulmonary insults. This work begins to address the need for mesenchyme-free culture conditions that permit transplantation of lineage-restricted and expandable AT2 organoids, while highlighting the feasibility of adoptive transfer of alveolar progenitors for future therapies. 

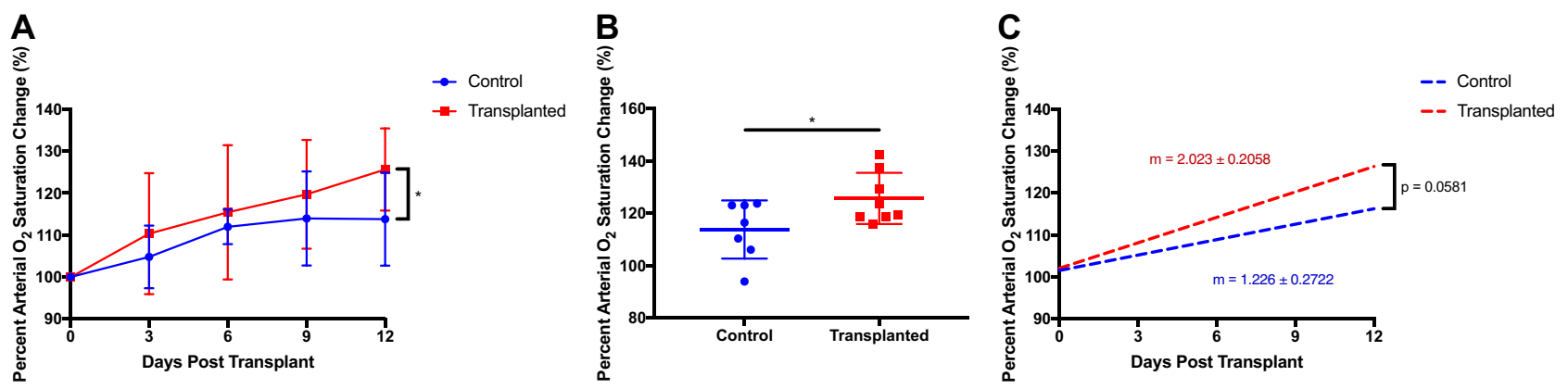

Fig. 5 Primary AT2 transplants improve pulmonary function post flu. a, b Pulse oximetry readings were taken from primary AT2- and mocktransplanted mice over the course of 12 days post transplant. A significant increase in percent change of $\% \mathrm{O}_{2}$ was detected at day 12 post transplant. c Linear regression of pulse oximetry data reveals an increasing trend in the slope of recovery of lung function in primary AT2 recipient mice. Data for $\mathbf{f}-\mathbf{h}$ is based on control $n=7$ mice, transplanted $n=8$ mice from four independent experiments. Error bars represent SD. ${ }^{*} p<0.05$ by two-way ANOVA. Linear regression slope $p$-value based on ANCOVA

\section{METHODS}

Animals and treatment

8- to 10-week-old mice were used for all experiments with males and females in roughly equal proportions. Experimenters were not blinded to

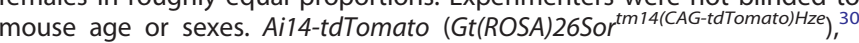
dsRed (Tg(CAG-DsRed $\left.{ }^{*} M S T\right) 1$ Nagy) ${ }^{31}$ UBI-GFP (Tg(UBC-GFP)30SCha) ${ }_{1}^{32}$ R26R-

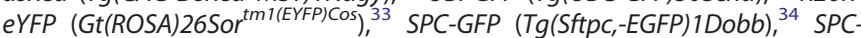

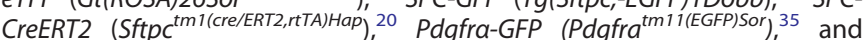
Wnt2-CreERT $2^{36}$ mice have been previously described. All studies were approved by the University of Pennsylvania's Institutional Animal Care and Use Committees, protocol 806262, and followed all NIH Office of Laboratory Animal Welfare regulations.

\section{Influenza infection}

Control and infected mice were first anesthetized using 3.5\% isoflurane in $100 \% \mathrm{O}_{2}$ via an anesthesia vaporizer system. Mice were intranasally administered 40-70 U TCID50 of influenza A/H1N1/Puerto Rico/8/34 (PR8) by pipetting $30 \mu \mathrm{L}$ of virus dissolved in phosphate-buffered saline (PBS) onto the nostrils of anesthetized mice in visually confirmed agonal breathing. Control mice were administered $30 \mu \mathrm{L}$ PBS using the same method. Only infected mice that lost $\geq 15 \%$ of their starting body weight by $11 \mathrm{DPI}$ were considered to be adequately infected and were used for all experiments involving influenza infection. Mouse weights were tracked by measuring at days $0,3,6,9,14,17,20$, and 23 post infection. Cells were transplanted at day 11 post infection.

\section{Acid injury}

Mice were anesthetized by intraperitoneal injections of $100 \mu \mathrm{L}$ ketamine/ xylazine $(100 \mathrm{mg} / \mathrm{kg})$. Sedated mice were intubated with a $20 \mathrm{G}$ angiocatheter (BD) as previously described. ${ }^{37}$ Mice were then placed in the right lateral recumbent position and a polyethylene 10 (PE10) catheter (Clay Adams) was directed into the right main stem bronchus, while pressure was applied to the left lung. Injury was induced by instilling $2 \mu \mathrm{L} /$ $\mathrm{g}$ of osmotically balanced $0.1 \mathrm{~N} \mathrm{HCl}$ into the right lung (maximum dose $=$ $50 \mu \mathrm{L}$ ) through the PE10 catheter as previously described. ${ }^{38}$ Cells were transplanted at day 1 post acid administration.

\section{Bleomycin administration}

For bleomycin-induced lung injury, we utilized intranasal administration as described for influenza infection with the following modifications. Mice received between 1.5 and $2.25 \mathrm{mg} / \mathrm{g}$ body weight bleomycin sulfate (13877-10, Cayman Chemicals) in a total volume of $50 \mu \mathrm{L}$ PBS. Given the inherent variability of bleomycin injury, this range of doses was used to ensure a cohort of mice that was sufficiently injured (lost $\geq 10 \%$ body weight) but survived the injury. Cells were transplanted at day 10 post bleomycin administration.

\section{S. pneumonia infection}

Sp strain TIGR4 (serotype 4) was grown in tryptic soy broth for 2 hours as previously described. ${ }^{39}$ Mice to be infected were anesthetized by intraperitoneal injections of $100 \mu \mathrm{L}$ ketamine/xylazine $(100 \mathrm{mg} / \mathrm{kg})$ and inoculated intranasally with $30 \mu \mathrm{L}$ of $5.3 \times 10^{7}$ CFU Sp suspension. Cells were transplanted 2 days post $S p$ administration.

\section{Organoid culture}

$\mathrm{CD}^{-} 5^{-} \mathrm{EPCAM}^{+} \beta 4^{-} \mathrm{AT} 2$ cells used for organoid culture experiments were fluorescence-activated cell (FACS) sorted from C57BL6, SPC-GFP, UBI-GFP, or dsRed mice. For each growth condition, 80,000 cells were seeded onto $90 \mu \mathrm{L}$ of Matrigel (BD) in a Corning 96 -well clear polystyrene flat-bottom microplate (Millipore Sigma). Organoid culture reagents used are as follows and each culture condition combination in addition to final in-solution concentrations of growth factors can be found in Table 1: $5 \%$ charcoaltreated fetal bovine serum (FBS) (Life Technologies), penicillin-streptomycin (P/S) solution (ATCC), SABM ${ }^{T M}$ Basal Medium (Lonza) + SAGM $^{\mathrm{TM}}$ SingleQuots ${ }^{\mathrm{TM}}$ Supplement Pack (Lonza) excluding hydrocortisone, gentamycin solution (Millipore Sigma), A83-01 (Millipore Sigma), recombinant murine Wnt3a (Peprotech), recombinant murine Noggin (Peprotech), recombinant human FGF10 (Peprotech), recombinant human KGF (FGF7, Peprotech), recombinant murine EGF (Peprotech), recombinant human Rspondin-1 (Peprotech), Y-27632 dihydrochloride (Stemcell Technologies), B27 $7^{\mathrm{TM}}$ supplement $(50 \times$, Thermo Fisher Scientific), GlutaMAX ${ }^{\mathrm{TM}}$ supplement $(100 \times$, Thermo Fisher Scientific), HEPES buffer solution (1 M, Thermo Fisher Scientific), and N2 supplement (100 $x$, Thermo Fisher Scientific). Organoids cultured in all conditions were grown in a $37^{\circ} \mathrm{C}$ incubator for 13 days without passaging and with media changes on day 5 and day 9 before collecting for RNA or transplant.

\section{Organoid diameter quantification}

Organoids grown from all mice listed in "Organoid culture" were used for diameter quantification. Tilescans of organoid-containing wells were imaged using the bright-field setting on a Leica DMi8 Microscope using a Leica DFC9000 sCMOS camera and Leica Application Suite X (LASX) software. Organoids were always grown in at least technical duplicates and all technical replicate wells were imaged and quantified. Diameter measurement was performed by using the built-in "Draw scalebar" function on LASX. Ten to 50 organoids were measured and averaged in each technical replicate, and technical replicates were averaged for a final biological replicate, consisting of $>30$ organoids measured across all technical replicates. The resultant diameter from each biological replicate was then normalized to the average diameter of $\mathrm{C} 1$ organoids, which were grown as a control in at least one technical replicate for all experiments. Reported average diameter for each condition comes from at least $n=3$ biological replicates.

\section{Organoid and primary cell transplants}

dsRed mice bred to a C57BL6 background were used to grow donor organoids for organoid transplant experiments. Organoid transplant recipients received 20,000 - 150,000 AT2 organoid cells grown in C2 and C12 for 13 days with media changes on days 5 and 9. UBI-GFP, SPC-GFP, or SPC-CreERT2(Ai14-tdTomato) mice bred to a C57BL6 background were used as donors for transplant experiments. SPC-CreERT2(Ai14-tdTomato) mice were given three doses of $0.25 \mathrm{mg} / \mathrm{g}$ body weight tamoxifen dissolved in $50 \mu \mathrm{L}$ corn oil every other day for 6 days. Mice given tamoxifen were killed 
for FACS 1 week following the final dose. Primary AT2 recipients received $900,000 \mathrm{CD}^{-} 5^{-}$EPCAM $^{+} \beta 4^{-}$AT2 cells. All recipients of both organoid and primary cell transplants were C57BL6. Recipient mice anesthetized with $3.5 \%$ isoflurane in $100 \% \mathrm{O}_{2}$ via an anesthesia vaporizer system were intranasally administered cells by pipetting single-cell suspension in PBS + $1 \% \mathrm{P} / \mathrm{S}$ onto the nostrils of anesthetized mice in visually confirmed agonal breathing.

\section{Collecting organoids for RNA and transplants}

Organoids were treated with $15 \mathrm{U} / \mathrm{mL}$ dispase II (Roche) in Hank's balanced salt solution (HBSS) for 30 minutes, rinsed with 2 mM EDTA (Thermo Fisher Scientific), incubated in $2 \mathrm{mM}$ EDTA for $5 \mathrm{~min}$ at $37^{\circ} \mathrm{C}$, mechanically dissociated by pipetting 50-100 times with a p200, and pelleted at $550 \times g$ for 5 minutes at $4{ }^{\circ} \mathrm{C}$. Cell pellets to be used for RNA were frozen by first aspirating supernatant followed by direct placement in a $-80^{\circ} \mathrm{C}$ freezer. Cell pellets to be used for organoid transplant had their supernatant aspirated and were resuspended in $30 \mu \mathrm{LBS}+1 \% \mathrm{P} / \mathrm{S}$ per number of cells to be transplanted. Resuspended organoid transplant cells were kept on ice between final resuspension and transplantation.

\section{Preparation of primary AT2 cells for transplant}

Directly after sorting, primary AT2 cells were pelleted at $550 \times g$ for 5 minutes at $4{ }^{\circ} \mathrm{C}$. After aspirating the supernatant, the cell pellet was resuspended in $30 \mu \mathrm{L}$ PBS $+1 \%$ P/S per 900,000 cells to be transplanted. Resuspended primary AT2 cells were kept on ice between final resuspension and transplantation.

\section{Pulse oximetry}

Repeated measurements of peripheral oxygen saturation $\left(\mathrm{SpO}_{2}\right)$ were taken using a MouseOx Plus Rat \& Mouse Pulse Oximeter and a MouseOx small collar sensor (Starr Life Sciences Corp.). Mice were shaven around the neck and shoulders where the collar sensor sits. Recordings were taken using MouseOx Premium Software (Starr Life Sciences Corp., Oakmont, PA, USA). Measurements were taken continuously for $>3$ minutes at a measurement rate of $15 \mathrm{~Hz}$. Measurements were imported into Microsoft Excel and all readings with a non-zero Error Code were filtered out. The average of these error-free readings was used to calculate the $\mathrm{SpO}_{2}$ reading for each mouse for each given time point. $\mathrm{SpO}_{2}$ measurements were normalized to pretreatment $\mathrm{SpO}_{2}$ values at day 0 .

\section{Lung tissue preparation for immunostaining}

Following sacrifice via isoflurane overdose, lungs were inflated at a constant pressure of $25 \mathrm{~cm} \mathrm{H}_{2} \mathrm{O}$ with $3.2 \%$ paraformaldehyde (PFA) for 30 minutes followed by incubation in 3.2\% PFA for another 30 minutes at room temperature. Fixed lungs were then washed in multiple PBS washes over the course of 1 hour at room temperature, followed by an overnight incubation in $30 \%$ sucrose shaking at $4{ }^{\circ} \mathrm{C}$, and then a 2 hour incubation in $15 \%$ sucrose $50 \%$ OCT compound (Fisher HealthCare) at room temperature. Finally, fixed lungs were embedded in OCT by flash freezing with dry ice and ethanol.

\section{Organoid preparation for cytospins}

Organoids were collected by first washing each organoid well with PBS, then scraping the Matrigel plug and organoids into a $15 \mathrm{~mL}$ tube using a p20 pipet tip. Each well was washed once more with PBS and all washes were collected in their respective tubes. Each tube was topped off with $10 \mathrm{~mL}$ cold PBS and the organoids in suspension were gently pipetted up to ten times with a p1000 to break up the Matrigel. Organoids were pelleted at $200 \times g$ for 5 minutes at $4{ }^{\circ} \mathrm{C}$, resuspended in $10 \mathrm{~mL}$ cold PBS, and shaken at room temperature on ice for $30 \mathrm{~min}$. Organoids were once more gently pipetted up to ten times to break up the remaining Matrigel before pelleting organoids again at $200 \times g$ for 5 minutes at $4{ }^{\circ} \mathrm{C}$. Organoids were resuspended in $10 \mathrm{~mL}$ cold $3.2 \%$ PFA and shaken at room temperature on ice for $30 \mathrm{~min}$. Organoids were pelleted once more at $200 \times g$ for 5 minutes at $4{ }^{\circ} \mathrm{C}$.

\section{Cytospins}

Sorted cells were pelleted at $550 \times g$ and organoids at $200 \times g$ for 5 minutes at $4{ }^{\circ} \mathrm{C}$ before resuspension in $200 \mu \mathrm{L} 3.2 \%$ PFA per number of slides to be cytospun. $200 \mu \mathrm{L}$ of resuspended cells or organoids was loaded into cytospin chambers. Cells were spun at 75 r.p.m. for 4 minutes and organoids were spun at 200 rpm for 5 minutes on a Cytospin 2 (Shandon).

\section{Immunostaining}

Seven-micrometer sections were cut on a Leica CM3050 S Research Cryostat (Leica Biosystems). Tissue sections and cytospins were further fixed for 5 minutes in 3.2\% PFA, rinsed three times with PBS, and blocked in blocking solution (PBS $+1 \%$ bovine serum albumin (Affymetrix) $+5 \%$ normal donkey serum (Jackson Immuno Research) $+0.1 \%$ Triton X-100 (Millipore Sigma) $+0.02 \%$ sodium azide (Millipore Sigma)) for $>30$ minutes. Slides were incubated in primary antibodies (listed below) in blocking solution overnight at $4{ }^{\circ} \mathrm{C}$. Slides were then washed three times with PBS $+0.1 \%$ Tween-20 (Millipore Sigma) and subsequently incubated with secondary antibodies (listed below) for $>2 \mathrm{~h}$ at room temperature. Slides were then washed once more with PBS $+0.1 \%$ Tween-20 prior to incubation in $1 \mu \mathrm{M}$ DAPI (Life Technologies) for 5 minutes, rinsed with PBS, and mounted with either Prolong Gold (Life Sciences) or Fluoroshield (Millipore Sigma). The following primary antibodies were used: rabbit antiSPC (1:2000, Millipore), rat anti-Lamp3 (1:500, Novus, clone 1010E1.01), rat anti-RAGE (1:500, R\&D, clone 175410), rabbit anti-Aqp5 (1:100, Abcam, clone EPR3747), Syrian hamster anti-Pdpn (1:100, DSHB, clone 8.1.1), rabbit anti-Krt5 (1:1000, BioLegend, clone Poly19055), chicken anti-Krt5 (1:500, BioLegend, clone Poly9059), goat anti-Scgb3a2 (1:200, R\&D, clone AF3465), and sheep anti-eGFP (1:500, Invitrogen, 10396164). The following secondary antibodies were used: Alexa Fluor ${ }^{\mathrm{TM}}$ 488-conjugated donkey anti-sheep (1:1000, Thermo Fisher Scientific), Alexa Fluor ${ }^{\mathrm{TM}}$ 488-conjugated donkey anti-rabbit (1:1000, Thermo Fisher Scientific), Alexa Fluor ${ }^{\text {TM }} 488$ donkey anti-rat (1:1000, Thermo Fisher Scientific), donkey anti-chicken Alexa Fluor ${ }^{\mathrm{TM}} 488$ (1:500, Jackson Immuno Research), fluorescein isothiocyanate-conjugated goat anti-chicken (1:1000, BioLegend, Poly24108), Alexa Fluor ${ }^{\mathrm{TM}}$ 568-conjugated donkey anti-rabbit (1:1000, Thermo Fisher Scientific), Alexa Fluor ${ }^{\mathrm{TM}}$ 568-conjugated donkey anti-goat (1:1000, Thermo Fisher Scientific), Alexa Fluor ${ }^{\mathrm{TM}}$ 647-conjugated donkey anti-rabbit (1:1000, Thermo Fisher Scientific), Alexa Fluor ${ }^{\mathrm{TM}}$ 647-conjugated donkey anti-goat (1:1000, Thermo Fisher Scientific), Alexa Fluor ${ }^{\mathrm{TM}}$ 647conjugated chicken anti-rat (1:1000, Thermo Fisher Scientific).

\section{Fluorescence-activated cell sorting}

Lung cells were isolated by first inflating lungs with $15 \mathrm{U} / \mathrm{mL}$ dispase II in HBSS (Thermo Fisher Scientific), tying off the trachea, and cutting lobes away from the main stem bronchi. Lobes were then incubated in dispase for $\mathbf{4 5}$ minutes shaking at room temperature and mechanically dissociated by pipetting in sort buffer (SB; Dulbecco's modified Eagle's medium (DMEM) (Thermo Fisher Scientific) $+2 \%$ cosmic calf serum (CC; Thermo Fisher Scientific) $+1 \% \mathrm{P} / \mathrm{S}$ ). After pelleting at $550 \times g$ for 5 minutes at $4{ }^{\circ} \mathrm{C}$, whole-lung suspension was treated with Red Blood Cell Lysis Buffer (Millipore Sigma) for 5 minutes, pelleted, and resuspended in SB + 1:1000 DNase I (Millipore Sigma) for a 45 minutes recovery period shaking at $37^{\circ} \mathrm{C}$. Whole-lung suspension was then repelleted and resuspended in $\mathrm{SB}+1: 50$ TruStain FcX (anti-mouse CD16/32) Antibody (BioLegend) for a 10 minutes blocking period at $37^{\circ} \mathrm{C}$. For sorts from C57BL6 mice, AT2 cells were collected using allophycocyanin (APC)/Cy7-conjugated rat antimouse CD45 antibody (1:200, BioLegend, 30-F11), Alexa Fluor ${ }^{\oplus}$ 488conjugated rat anti-mouse CD326 (EpCAM) antibody (1:200, BioLegend, G8.8), and phycoerythrin (PE)-conjugated rat anti-mouse CD104 (integrin $\beta 4$ ) antibody $(1: 100$, BioLegend, 346-11A). For sorts from mice expressing a GFP fluorophore, AT2 cells were collected using the C57BL6 sort scheme above but with PE-conjugated rat anti-mouse EpCAM (1:200, BioLegend, G8.8) and APC-conjugated rat anti-mouse integrin $\beta 4$ antibody (1:100, BioLegend, 346-11A) instead of the respective EpCAM and integrin $\beta 4$ antibodies listed above. For sorts from mice expressing a RFP fluorophore, AT2 cells were collected using the C57BL6 sort scheme above but with APC-conjugated rat anti-mouse integrin $\beta 4$ antibody instead of the respective integrin $\beta 4$ antibody listed above. Lung mesenchyme collected from all aforementioned mice was collected by adding APC/Fire ${ }^{\mathrm{TM}} 750$ conjugated rat anti-mouse CD31 (PECAM) (1:200, BioLegend, MEC13.3) to the sort scheme to purify CD45- CD31- EpCAM- mesenchymal cells. Staining was performed for 45 minutes at $4{ }^{\circ} \mathrm{C}$, followed by a final spindown at $550 \times g$ for 5 minutes at $4^{\circ} \mathrm{C}$. Stained cells and fluorescence minus one controls were then resuspended in SB + 1:1000 DNase + 1:1000 Draq7 (Beckman Coulter) as a live/dead stain. All FACS sorting was done on a BD FACSJazz (BD Biosciences) and cells were collected in Falcon ${ }^{\mathrm{TM}}$ 
round-bottom polystyrene tubes (Thermo Fisher Scientific) in $300 \mu \mathrm{L}$ $\mathrm{DMEM}+20 \% \mathrm{CC}+2 \% \mathrm{P} / \mathrm{S}$.

\section{Quantitative reverse transcriptase PCR}

RNA was isolated from both organoids and sorted cells using a ReliaPrep ${ }^{\mathrm{TM}}$ RNA Cell Miniprep kit (Promega). The amount of RNA input for cDNA synthesis was standardized within each experiment to the RNA isolate with the lowest concentration as measured by Nanodrop (Thermo Fisher Scientific). cDNA was synthesized using iScript ${ }^{\mathrm{TM}}$ Reverse Transcription Supermix (BioRad). Primers used are as follows: SPC, forward $5^{\prime}$ ATGGACATGAGTAGCAAAGAGGT-3', reverse 5'-CACGATGAGAAGGCGTT GAG-3'; Krt5, forward 5'-TCCAGTGTGTCCTTCCGAAGT-3', reverse $5^{\prime}$ TGCCTCCGCCAGAACTGTA-3'; Scgb3a2, forward 5'-CCACTGCCCTTCTCAT CAACC- $3^{\prime}$, reverse $5^{\prime}$-TGTCGTCCAAAGGTACAGGTA-3'; $L 19$, forward $5^{\prime}$ ATGTATCACAGCCTGTACCTG-3', reverse $5^{\prime}$-TTCTTGGTCTCTTCCTCCTTG- ${ }^{\prime}$. Expression of each gene is relative to expression of $\mathrm{L} 19$ within that sample. qPCR was run on an Applied Biosystems QuantStudio 6 Real-Time PCR System (Thermo Fisher Scientific).

\section{Statistics}

All statistical tests were performed using GraphPad Prism 7. P-values were calculated using unpaired two-tailed $t$-tests with Welch's correction or ordinary one-way or two-way analysis of variance for comparisons involving multiple variables. Comparison of slopes from linear regression was calculated using an equivalent test to analysis of covariance in Chapter 18 of ref. ${ }^{40}$

\section{Reporting summary}

Further information on research design is available in the Nature Research Reporting Summary linked to this article.

\section{DATA AVAILABILITY}

The data that support the findings of this study are available from the corresponding author upon reasonable request.

\section{ACKNOWLEDGEMENTS}

We thank Chetan Rane, the CHOP Flow Cytometry Core, and the Penn Cardiovascular Institute (CVI) Histology and Gene Expression Core for their assistance in performing these studies. We acknowledge clker.com for their free public domain vector mouse graphics and the CDC's Seasonal Influenza Resource Center for their free influenza graphic (https://www.cdc.gov/flu/resource-center/freeresources/index.htm) used in this article.

\section{AUTHOR CONTRIBUTIONS}

A.I.W. and A.E.V. designed the experiments and wrote the manuscript. A.E.V. and E.E. M. supervised experimental design. A.I.W., S.R.J., G.Z., K.Q., J.F., K.N., D.C.L., E.Q.L., A.J.P. and G.S.W. performed experiments. A.I.W. acquired data and performed data analyses.

\section{ADDITIONAL INFORMATION}

Supplementary information accompanies the paper on the npj Regenerative Medicine website (https://doi.org/10.1038/s41536-019-0080-9).

Competing interests: The authors declare no competing interests.

Publisher's note: Springer Nature remains neutral with regard to jurisdictional claims in published maps and institutional affiliations.

\section{REFERENCES}

1. Evans, M. J., Cabral, L. J., Stephens, R. J. \& Freeman, G. Renewal of alveolar epithelium in the rat following exposure to NO2. Am. J. Pathol. 70, 175-198 (1973).

2. Barkauskas, C. E. et al. Type 2 alveolar cells are stem cells in adult lung. J. Clin. Invest 123, 3025-3036 (2013).

3. Vaughan, A. E. et al. Lineage-negative progenitors mobilize to regenerate lung epithelium after major injury. Nature 517, 621-625 (2015).
4. Rane, C. K. et al. Development of solitary chemosensory cells in the distal lung after severe influenza injury. Am. J. Physiol. Lung Cell Mol. Physiol. https://doi.org/ 10.1152/ajplung.00032.2019 (2019).

5. Zuo, W. et al. p63(+)Krt5(+) distal airway stem cells are essential for lung regeneration. Nature 517, 616-620 (2015).

6. $\mathrm{Xi}, \mathrm{Y}$. et al. Local lung hypoxia determines epithelial fate decisions during alveolar regeneration. Nat. Cell Biol. 19, 904-914 (2017).

7. Yang, Y. et al. Spatial-temporal lineage restrictions of embryonic p63+ progenitors establish distinct stem cell pools in adult airways. Dev. Cell 44, 752-761 (2018). e754.

8. Liu, W., Peng, L., Liu, H. \& Hua, S. Pulmonary function and clinical manifestations of patients infected with mild influenza A virus subtype H1N1: a one-year followup. PLOS ONE 10, e0133698 (2015).

9. Koppe, S., Túlio, A. I. B., Villegas, I. L. P. \& Motter, A. A. Pulmonary function in patients with pandemic H1N1. Fisioter. Mov. 29, 805-812 (2016).

10. Chen, J. et al. Long term outcomes in survivors of epidemic Influenza A (H7N9) virus infection. Sci. Rep. 7, 17275 (2017).

11. Zacharias, W. J. et al. Regeneration of the lung alveolus by an evolutionarily conserved epithelial progenitor. Nature 555, 251-255 (2018).

12. Zepp, J. A. et al. Distinct mesenchymal lineages and niches promote epithelial self-renewal and myofibrogenesis in the lung. Cell 170, 1134-1148 (2017). e1110.

13. Tanjore, $\mathrm{H}$. et al. Contribution of epithelial-derived fibroblasts to bleomycininduced lung fibrosis. Am. J. Respir. Crit. Care Med. 180, 657-665 (2009).

14. Lee, D. W. et al. Creation of myocardial fibrosis by transplantation of fibroblasts primed with survival factors. Am. J. Physiol. Heart Circ. Physiol. 301, H1004-H1014 (2011).

15. Madala, S. K. et al. Bone marrow-derived stromal cells are invasive and hyperproliferative and alter transforming growth factor-alpha-induced pulmonary fibrosis. Am. J. Respir. Cell Mol. Biol. 50, 777-786 (2014)

16. Kim, K. K. et al. Alveolar epithelial cell mesenchymal transition develops in vivo during pulmonary fibrosis and is regulated by the extracellular matrix. Proc. Natl Acad. Sci. USA 103, 13180-13185 (2006).

17. Marmai, C. et al. Alveolar epithelial cells express mesenchymal proteins in patients with idiopathic pulmonary fibrosis. Am. J. Physiol. Lung Cell Mol. Physiol. 301, L71-L78 (2011).

18. Rock, J. R. et al. Multiple stromal populations contribute to pulmonary fibrosis without evidence for epithelial to mesenchymal transition. Proc. Natl Acad. Sci. USA 108, E1475-E1483 (2011).

19. Wolters, P. J., Collard, H. R. \& Jones, K. D. Pathogenesis of idiopathic pulmonary fibrosis. Annu. Rev. Pathol. 9, 157-179 (2014).

20. Chapman, H. A. et al. Integrin alpha6beta4 identifies an adult distal lung epithelial population with regenerative potential in mice. J. Clin. Invest. 121, 2855-2862 (2011).

21. Bellusci, S., Grindley, J., Emoto, H., Itoh, N. \& Hogan, B. L. Fibroblast growth factor 10 (FGF10) and branching morphogenesis in the embryonic mouse lung Development 124, 4867-4878 (1997).

22. Frank, D. B. et al. Emergence of a wave of Wnt signaling that regulates lung alveologenesis by controlling epithelial self-renewal and differentiation. Cell Rep. 17, 2312-2325 (2016).

23. Francis, K. P. et al. Visualizing pneumococcal infections in the lungs of live mice using bioluminescent Streptococcus pneumoniae transformed with a novel grampositive lux transposon. Infect. Immun. 69, 3350-3358 (2001).

24. Takebe, T. et al. Vascularized and functional human liver from an iPSC-derived organ bud transplant. Nature 499, 481-484 (2013).

25. Azuma, H. et al. Robust expansion of human hepatocytes in Fah-/-/Rag2-/-/II $\mathrm{rg}-/$ mice. Nat. Biotechnol. 25, 903-910 (2007).

26. Shackleton, $M$. et al. Generation of a functional mammary gland from a single stem cell. Nature 439, 84-88 (2006).

27. Fordham, R. P. et al. Transplantation of expanded fetal intestinal progenitors contributes to colon regeneration after injury. Cell Stem Cell 13, 734-744 (2013).

28. Sugimoto, S. et al. Reconstruction of the human colon epithelium in vivo. Cell Stem Cell 22, 171-176 (2018). e175.

29. Guo, L. et al. Interrupted reprogramming of alveolar type II cells induces progenitor-like cells that ameliorate pulmonary fibrosis. NPJ Regen. Med. 3, 14 (2018).

30. Madisen, L. et al. A robust and high-throughput Cre reporting and characterization system for the whole mouse brain. Nat. Neurosci. 13, 133-140 (2010).

31. Vintersten, K. et al. Mouse in red: red fluorescent protein expression in mouse ES cells, embryos, and adult animals. Genesis 40, 241-246 (2004).

32. Schaefer, B. C., Schaefer, M. L., Kappler, J. W., Marrack, P. \& Kedl, R. M. Observation of antigen-dependent CD8+ T-cell/ dendritic cell interactions in vivo. Cell Immunol. 214, 110-122 (2001).

33. Srinivas, S. et al. Cre reporter strains produced by targeted insertion of EYFP and ECFP into the ROSA26 locus. BMC Dev. Biol. 1, 4 (2001). 
34. Vanderbilt, J. N. et al. High-efficiency type II cell-enhanced green fluorescent protein expression facilitates cellular identification, tracking, and isolation. Am. J. Respir. Cell Mol. Biol. 53, 14-21 (2015).

35. Hamilton, T. G., Klinghoffer, R. A., Corrin, P. D. \& Soriano, P. Evolutionary divergence of platelet-derived growth factor alpha receptor signaling mechanisms. Mol. Cell Biol. 23, 4013-4025 (2003).

36. Peng, $\mathrm{T}$. et al. Coordination of heart and lung co-development by a multipotent cardiopulmonary progenitor. Nature 500, 589-592 (2013).

37. Das, S., MacDonald, K., Chang, H. Y. \& Mitzner, W. A simple method of mouse lung intubation. J. Vis. Exp. e50318. https://doi.org/10.3791/50318 (2013).

38. Paris, A. J. et al. Using selective lung injury to improve murine models of spatially heterogeneous lung diseases. PLOS ONE 14, e0202456 (2019).

39. Wang, Y. et al. Cross-protective mucosal immunity mediated by memory Th17 cells against Streptococcus pneumoniae lung infection. Mucosal Immunol. 10, 250-259 (2017).

40. Zar, J. H. Biostatistical Analysis. 2nd edn (Prentice-Hall, 1984).
Open Access This article is licensed under a Creative Commons Attribution 4.0 International License, which permits use, sharing, adaptation, distribution and reproduction in any medium or format, as long as you give appropriate credit to the original author(s) and the source, provide a link to the Creative Commons license, and indicate if changes were made. The images or other third party material in this article are included in the article's Creative Commons license, unless indicated otherwise in a credit line to the material. If material is not included in the article's Creative Commons license and your intended use is not permitted by statutory regulation or exceeds the permitted use, you will need to obtain permission directly from the copyright holder. To view a copy of this license, visit http://creativecommons. org/licenses/by/4.0/.

(c) The Author(s) 2019 